\title{
Analysis and calculation of dimension of gauge block in sine bar measuring taper angle
}

\author{
Ping Kan, Miao He \\ Sichuan Information Technology College, China \\ fjjscitc@163.com
}

Keywords: sine bar, measure, taper angle, dimension of gauge block

\begin{abstract}
The sine bar is a precision measuring tool which is used for indirect measurement or machining of precision angle by the principle of sine function. When the cone taper angle is measured by sine bar, which is at one end of the sine bar need to pad the size of the corresponding block below it, the sine bat will be risen and the angle of lifting is related to the type and the method of the measuring cone. The dimension of gauge block is decided by the angle of lifting and sine function. The error of Block size will directly affect the accuracy of measurement results.
\end{abstract}

\section{Introduction}

There are several measuring methods of the taper angle of the cone, such as coloring, cylinder block, two groups of cylinders with different diameters, two spheres of different diameter measurement within the taper angle of the cone, two different diameter of the ball and the gauge block measurement taper angle, sine bar measuring taper angle. The sine bar measuring the angle of the cone is commonly.

The sine bar is a precision measuring tool which is used for indirect measurement or machining of precision angle by the principle of sine function. The sine bar Commonly is used to measuring the taper angle of the inner and outside cone and the head diameter of the cone, and can also measuring angle block angle, the angle between hole center line and a plane, and being a machining fixture of precision parts, especially which is the most common in the measurement of the taper angle. The sine bar can measure outside taper angle, inner taper angle, taper angle of the cylindrical cone, taper angle of the synthetic cone, taper angle of the different to cone, but the formulas of gauge which we use dimension of gauge block is different. If the dimension of gauge block size is wrong and it will directly affect the accuracy of the measurement results, so we need to clear each measurement of the dimension of gauge block size.

\section{The principle of measure ment}

The sine bar is divided into two kinds of narrow and wide, and according to the structure of each type in two cylinder center distance is divided into two kinds of $100 \mathrm{~mm}$ and $200 \mathrm{~mm}$. sine bar structure in which the lower end has a diameter equal to two and two cylindrical center cylindrical connection parallel the upper surface of the working face. Thus, as shown in Figure 1, we can see $\alpha=\alpha_{1}$ by the geometrical knowledge. Based on the principle of trigonometric functions, we can see $\sin \alpha=\frac{C O_{1}}{O O_{1}}=\frac{B O_{1}-B C}{O O_{1}}=\frac{h+R-R}{L}=\frac{h}{L}$ 
So we can know $\sin \alpha=\frac{\boldsymbol{h}}{\boldsymbol{L}}$

In equation $1-1, \alpha$ is the nominal value of the taper angle of the workpiece $\left(^{\circ}\right)$, and $\mathrm{L}$ is two cylinder center distance of the sine bar $(\mathrm{mm})$.

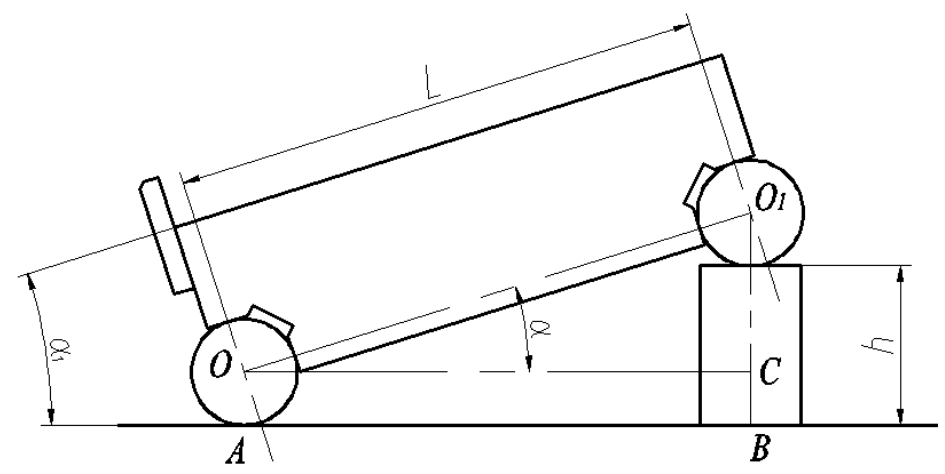

Fig 1.measurement principle of sine bar

Equation 1-1 only illustrates measurement principle of sine bar measuring taper angle and the relationship between the dimension of gauge block and the taper angle. When we calculate the dimension of gauge block of the sine bar using, firstly, according to the type of cone taper angle of the actual sine bar measuring and geometric relationships, we must rightly find the angle to be lifted of the sine bar, and secondly we need to be calculated by trigonometric functions and determined the dimension of gauge block size. In short, the dimension of gauge block size is chosen by the measured actual situation.

\section{Different types of cone tape $r$ angle of measure ment}

When several different types of taper angle are measured by sine bar, because of the different between test methods and methods for measuring the cone place, so the pad method for determining the dimension of gauge block sizes are different. In fact, we should compute taper angle in accordance with cone type, and it's critical to be able to correctly place the test cone, and then determine the taper angle by geometry knowled ge.

The measuring method is shown in figure 2 that the sine bar measure outside cone taper angle. The large end of the cone is leaned against the side fence, and the cylinder below the sine bar of the small end of the pad position desire gauge blocks which needed. In this case, the $\alpha$ angle is equal to the cone taper angle. According to trigonometric formulas, the pad gauge block size $h$ is, $h=L \sin \alpha$.

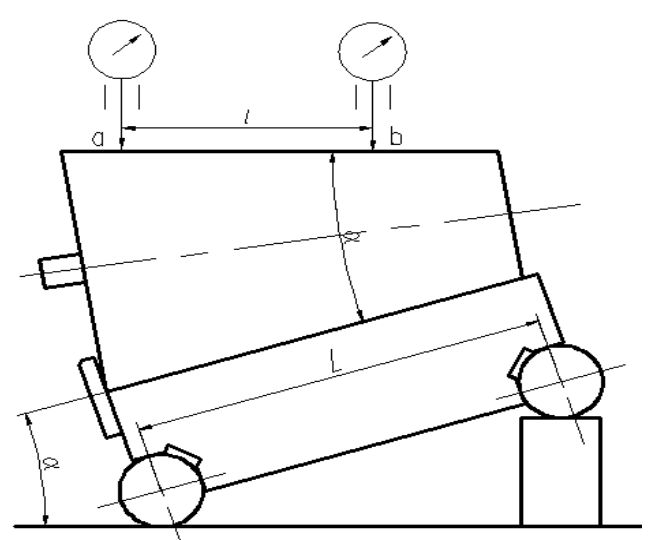

Fig 2.outside cone taper angle of measurement 
The measuring method is shown in figure 3 that the sine bar measure inner cone taper angle. Figure $\mathrm{a}$ is a chart which is a generatrix of measurement taper angle of inner cone, and Figure $\mathrm{b}$ is a chart which is a generatrix of measurement taper angle of inner cone. However, If the inner cone different places are measured, the placement are also different. As shown in Figure 3, according to the knowledge of geometry, we easily find the relationship between the corresponding angle, then the angle of the lift sine bar of the measuring is half taper angle of the cone, which is that the lift angle of the sine bar is half of measuring cone taper angle. So the pad gauge block size $h$ is,

$$
h=L \sin \frac{\alpha}{2} \text {. }
$$
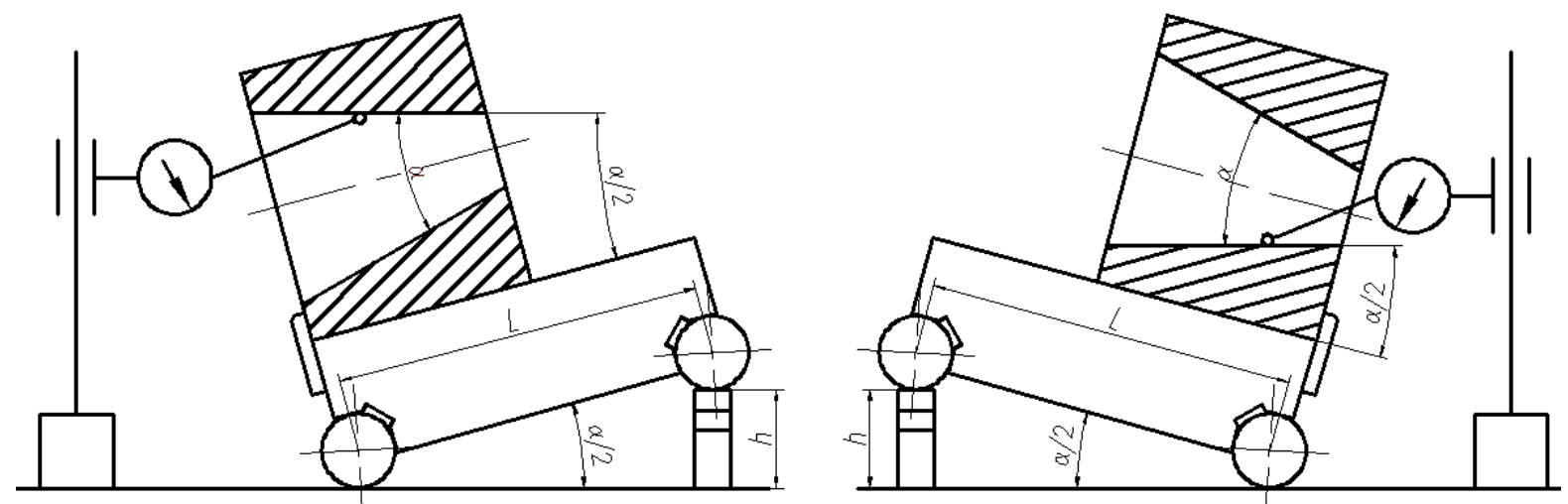

Fig 3. Inner cone taper angle of measurement

The measuring method is shown in figure 4 that outside cone taper angle is measured by top sine bar. The cylinder below the sine bar of the small end of the pad position desire gauge blocks which needed, and lift sine bar of the angle of the measuring is taper angle of the cone. So the pad gauge block size $\mathrm{h}$ is, $h=L \sin \frac{\alpha}{2}$.

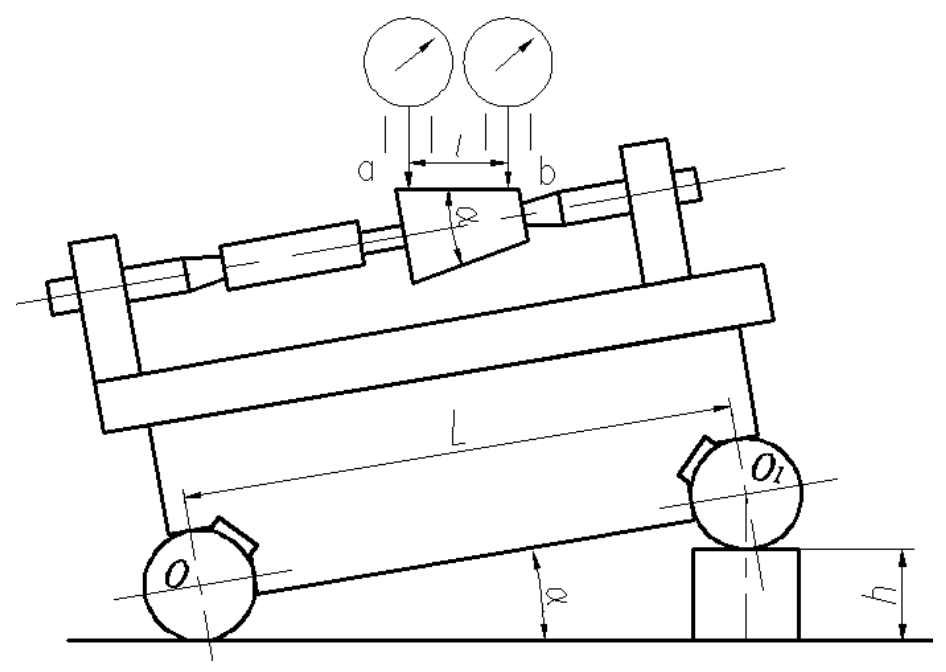

Fig 4.outside cone taper angle measured by top sine bar

The measuring method is shown in figure 5 that taper angle of the cylindrical cone is measured. Because the length of cylindrical cone axial is short, we use generatrix of cylinder surface to position to measure the taper angle of the cylindrical cone. As shown in Figure 3, One end of the 
cylinder is against the inner side of the tailgate of sine bar, and cone is raised, so the relationship between the angle is, $\alpha=\beta / 2$. So the pad gauge block size $\mathrm{h}$ is, $h=L \sin \alpha=L \sin \frac{\beta}{2}$.

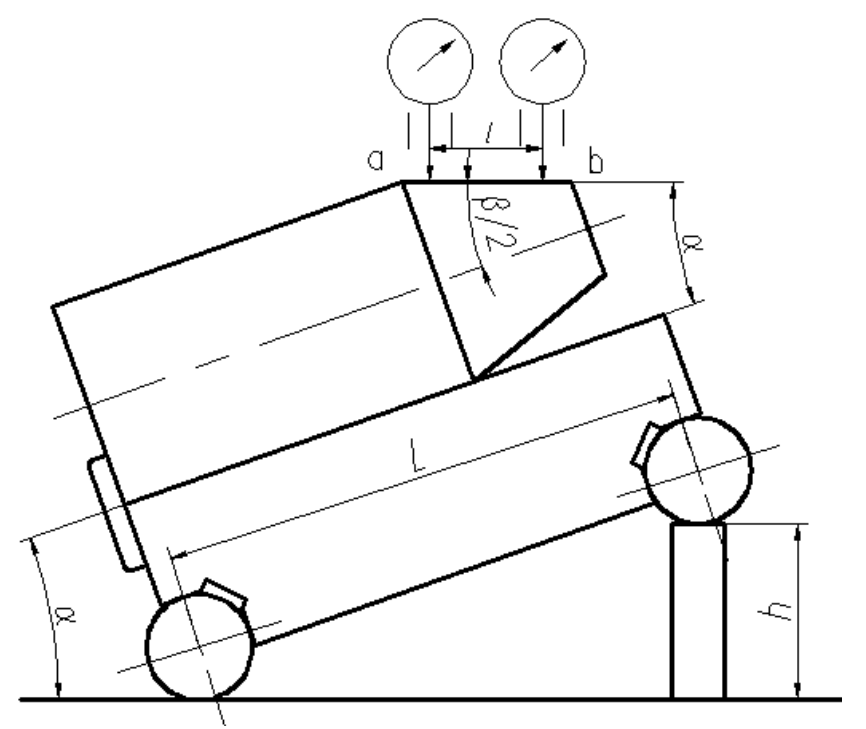

Fig 5.taper angle of the cylindrical cone of measurement

The measuring method is shown in figure 6(a) that taper angle of the cone is measured, whose cones are narrowed in the same direction. As shown in figure 6(a), if we measure the left side of the cone, the pad gauge block size $\mathrm{h}$ is equal to the Equation 2-1. However, if we measure the right side of the cone, we need new method. We are hypothesis that the right taper angle of the cone is $\beta$ and the left taper angle of the cone is $\boldsymbol{\theta}$. From the figure 6 , the angle of the lift sine bar of the measuring is nothing to do with $\alpha$ and $\beta$, and $\alpha=\beta / 2+\theta / 2$. So the pad gauge block size $\mathrm{h}$ is, $h=L \sin \alpha=L \sin \left(\frac{\beta}{2}+\frac{\theta}{2}\right)$.

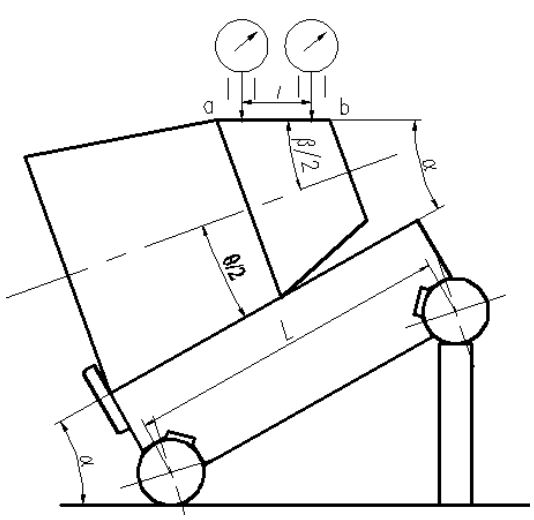

(a) in the same direction

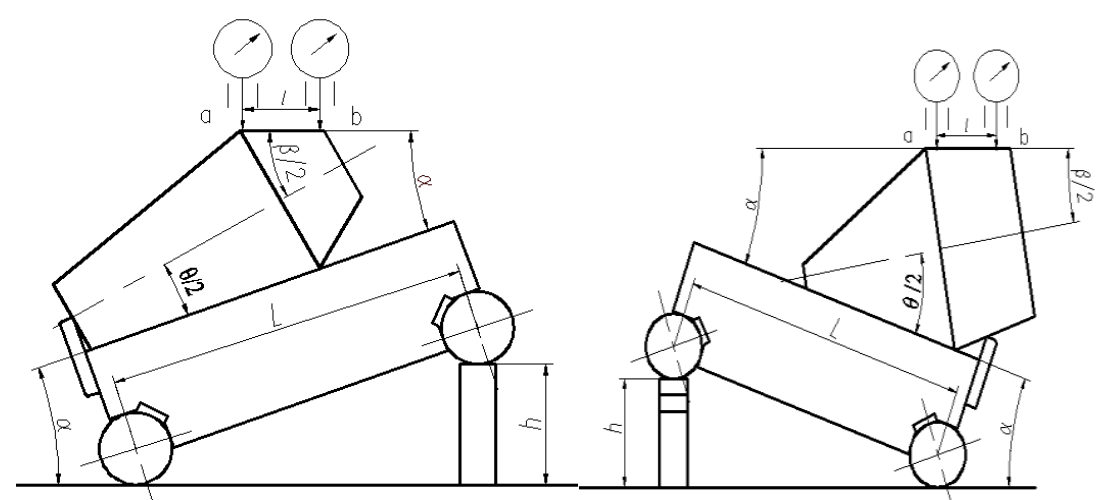

(b) $(\beta>\theta)$

(c) $(\beta<\theta)$

Fig 6. taper angle of the synthetic cone of measurement

The measuring method is shown in figure 6(b) that taper angle of the cone is measured, whose cones are narrowed in the other direction. We are hypothesis that the right taper angle of the cone is 
$\beta$ and the left taper angle of the cone is $\theta$. From figure7(b), If $\beta>\theta, \frac{\beta}{2}=\frac{\theta}{2}+\alpha$, and $\alpha=\frac{\beta}{2}-\frac{\theta}{2}$. So the pad gauge block size h is, $h=L \sin \alpha=L \sin \left(\frac{\beta}{2}-\frac{\theta}{2}\right)$.

From figure 6(c), If $\beta<\theta, \frac{\theta}{2}=\frac{\beta}{2}+\alpha$, and $\alpha=\frac{\theta}{2}-\frac{\beta}{2}$. So the pad gauge block size $\mathrm{h}$ is, $h=L \sin \alpha=L \sin \left(\frac{\theta}{2}-\frac{\beta}{2}\right)$

\section{Summary}

When we measure taper angle with a sine bar, we need to pad with the corresponding of dimension of gauge block size. But the dimension of gauge block size is acquired, we need to know the angle of the lift sine bar. We measure different types of cones taper angle, because of the different methods of placing the cone, sine bar pad Block was raised different angles so that the size of dimension of gauge block have their respective formula. When cone taper angle is measured, we are based on the cone of the actual situation. Firstly, the angle of the lift sine bar is determined with geometric knowledge. Secondly, the formula is determined in each case by trigonometric function knowledge. Lastly, the pad gauge block size is determined. It is the first step of cone taper angle of measurement that the pad gauge block size is determined, and if it is wrong, it will directly affect the measurement accuracy of the results. It is very important that the pad gauge block size is computed in cone taper angle of measurement.

\section{REFERENCES}

[1].Zhizhen Lu. Mechanical measuring technology [M]. China Machine Press. 2013,P111-112, in Chinese.

[2].Xingfu Liu. Accurate and simple measurement method of the inner and outside taper angle [J]. Machinery Industry Standardization \& Quality .2007(1),P45-47, in Chinese.

[3].Wei Liu. On sine bar differential comparison rapid measurement of angle control size [J]. Hunan Agricultural Machinery.2012,39(1),P123,127, in Chinese.

[4].Chao Hong, Hongchou Liu, Ping Wu. Fitter processing method measuring in the digital age [J]. Metal Working. 2015(3), P33-34, in Chinese.

[5].Xingfu Liu. The method of measuring the inner taper angle by sine bar[J]. MACHINERY. 2002,40(10),P43-44, in Chinese.

[6].Minggen Xue. Tapered taper angle fast calibration method [J]. KEJI JINGJI SHICHANG. 2007(12), P170-171, in Chinese.

[7].Xingfu Liu. An Easy Way to Accurate Measurement of Inside and Outside Cone with Sine Protractor [J]. EQUIPMENT MANUFACTURING TECHNOLOGY. 2005(1), P28-29, in Chinese. [8].Zuohua Wu. Discussion on Method of Measuring Taper angle on Sine Protractor [J]. Metrology \& Measurement Technology. 2012,32(1), P36-38, in Chinese.

[9].Taotao Wang, Jianbo Kang. Sine bar measuring cone taper angle and uncertainty evaluation[J]. Journal of Henan Science and Technology. 2013(3), P92-93, in Chinese. 